\title{
The Characteristics of Soil Organic Nitrogen Using Efficiency in Different Sugarbeet Genotypes
}

\author{
Jian-Chao Zhou, Qiu-Hong Wang, Xiao-Chun Wang \\ Academy of Crop Science, Heilongjiang University, Harbin, China \\ Email address: \\ zhou88767@126.com (Jian-Chao Zhou),wangqiuhong119@126.com (Qiu-Hong Wang),wxc208282@163.com (Xiao-Chun Wang) \\ To cite this article: \\ Jian-Chao Zhou, Qiu-Hong Wang, Xiao-Chun Wang. The Characteristics of Soil Organic Nitrogen Using Efficiency in Different Sugarbeet \\ Genotypes. Agriculture, Forestry and Fisheries. Vol. 6, No. 4, 2017, pp. 145-148. doi: 10.11648/j.aff.20170604.16
}

Received: May 17, 2017; Accepted: July 12, 2017; Published: August 7, 2017

\begin{abstract}
The research into variation of soil organic nitrogen use efficiency and absorption efficiency with different sugarbeet genotypes provides a theoretical basis for the selection, cultivation and breeding of high-quality genotypes. In view of this, pot and field split-plot experiments have been conducted on 100 sugar beet genotypes under chamber and field conditions. The results were as follows: in growth chamber pot experiments, whole plant and root organic nitrogen use efficiency varied significantly among genotypes; higher soil organic nitrogen use efficiency at seedling stage preceded higher absorption capacity and organic nitrogen use efficiency during the following three growth stages; higher transformation quantity and soil organic $\mathrm{N}$ use efficiency predicted higher root-shoot ratio at harvest stage.
\end{abstract}

Keywords: Sugar Beet, Organic Nitrogen, Use Efficiency, Absorption Efficiency

\section{Introduction}

Sugar beet is one of the most important sugar crops in China, and $\mathrm{N}$ is the key factor of beet yield and quality [7, 15]. Soil organic $\mathrm{N}$ is the main source of soil $\mathrm{N}$ nutrient [16], which accounts for $90 \%$ of the soil total N [1]. Recent studies on soil organic $\mathrm{N}$ are centered more on mineralization of organic $\mathrm{N}[1,4,2]$ and the composition of soil organic $\mathrm{N}$, specially on amino-N [9] and aminosaccharide $\mathrm{N}$ [10]. However, considering the great demands of sustainable agriculture, better use and absorption of soil inherent $\mathrm{N}$, though long neglected, might represent a promising method for higher yield and quality [4]. Therefore, 100 different sugar beet genotypes have been compared and investigated for ideal varieties featuring high soil organic $\mathrm{N}$ use efficiency, which forms a theoretical basis for reasonable $\mathrm{N}$ fertilization and beet breeding.

\section{Materials and Methods}

\subsection{Materials}

According to the ploidy, germ number and genetic background, 100 different sugar beet genotypes were selected from the medium-term gene bank of sugar beet in China.

\subsection{Pot Experiment at Seedling Stage}

100 beet varieties were planted in plastic pots of treated soil inorganic $\mathrm{N}$ was leached by $0.01 \mathrm{~mol} \cdot \mathrm{L}^{-1} \mathrm{CaCl}_{2}$, and fertilized by $\mathrm{N}_{0}$ Hoagland nutrition solution [2], then grown in a growth chamber (50 days) with a condition of $25^{\circ} \mathrm{C}$ in daylight $\left(14 \mathrm{~h}, 200 \mu \mathrm{mol} \cdot \mathrm{m}^{-2} \cdot \mathrm{s}^{-1}\right)$ and $18^{\circ} \mathrm{C}$ in dark $(10 \mathrm{~h})$.

\subsection{Field Experiment}

Nylon net method was adopted in planting into culture frame respectively 1 high and 1 low varieties in field. The nylon net holes were $300 \#$ in diameter, 900 grams of soil in each net with a soil density $1.3 \mathrm{~g} / \mathrm{cm}^{3}$. These nets were left at the center of each culture frame and then planted.

\subsection{Data Processing}

All data was processed by Microsoft Excel 2007 and IBM SPSS Statistics 20.0 . 


\section{Results and Analysis}

\subsection{Variation of Organic Nitrogen Use Efficiency Among Sugar Beet Genotypes at Seedling Stage}

From the perspective of plants' nitrogen biological efficiency, the organic $\mathrm{N}$ use efficiency of the whole plants (ONUEp) and that of the roots (ONUEr) among the 100 genotypes of sugar beet were compared. Considerable difference in ONUE can be seen in Table 1, the variable coefficient of either ONUEp or ONUEr is over $10 \%$.

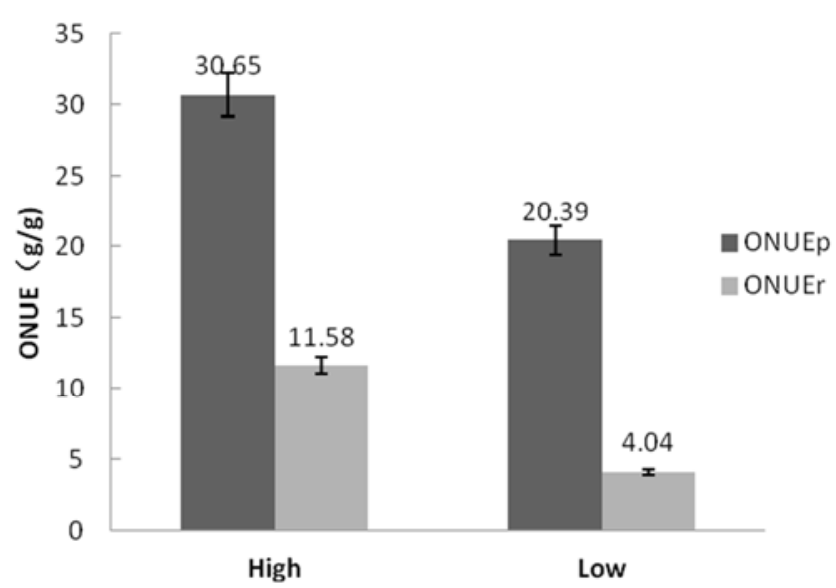

Figure 1. Difference between ONUEp or ONUEr of the Top 5 Genotypes and the last 5 genotypes of sugar beet.
Table 1. Difference between ONUEp and ONUEr of the 100 genotypes.

\begin{tabular}{lll}
\hline Plant Organ & ONUE $(\mathbf{g} / \mathbf{g})$ & CV $(\%)$ \\
\hline Whole plant(ONUEp) & $24.60 \pm 2.26$ & 21.19 \\
Root(ONUEr) & $8.27 \pm 0.86$ & 12.10 \\
\hline
\end{tabular}

ONUE is measured by efficiency of dry matter productivity. Figure 1 shows that the average ONUEp of the top 5 genotypes is $30.65 \mathrm{~g} / \mathrm{g}$, in contrast with $20.39 \mathrm{~g} / \mathrm{g}$ for the last 5 . The higher ONUEp is 1.5 times the lower one. While the average ONUEr of the top 5 genotypes is $11.58 \mathrm{~g} / \mathrm{g}$, in contrast with $4.04 \mathrm{~g} / \mathrm{g}$ for the last 5 . The higher ONUEr is 2.8 times the lower one.

\subsection{ONUEp for Different Genotypes of Sugar Beet During Each Development Stage}

Select one out of each representative genotype with high ONUEp (KWS8138) and low ONUEp (BETA176), and then conduct a further field research on their ONUEp during different development stages. Results show that ONUEp increases all the way from seedling stage, reaches its peak at vegetative stage, and then gradually goes down till the sugar accumulation stage (Table 2). From seedling to harvest, organic nitrogen use efficiency of the high ONUEp genotypes are considerably higher than that of the low ONUEp genotypes, which corresponds to the results of indoor pot experiment at seedling stage.

Table 2. ONUEp during the different developmental stages of Sugar beet $(g / g)$.

\begin{tabular}{lllll}
\hline Genotypes & Seedling stage & Vegetative stage & Root and sugar increasing stage & Sugar accumulation stage \\
\hline WS8138 (High Efficiency) & $21.12 \pm 2.33 \mathrm{a}$ & $24.48 \pm 2.68 \mathrm{a}$ & $22.11 \pm 2.14 \mathrm{a}$ & $12.51 \pm 1.12 \mathrm{a}$ \\
BETA176 (Low Efficiency) & $11.61 \pm 1.19 \mathrm{~b}$ & $15.98 \pm 1.07 \mathrm{~b}$ & $13.74 \pm 1.08 \mathrm{~b}$ & $5.74 \pm 0.68 \mathrm{~b}$ \\
\hline
\end{tabular}

Note: Values labeled with different letter in the same column means the difference between them has reached to 0.05 statistic level.

\subsection{Transformation Quantity of Soil Organic N During Sugar Beet Growth Season}

We can see in Table 3 that Soil Organic Nitrogen Transformation Quantity, for both selected groups of genotypes under test, is the lowest at seedling stage and highest at sugar accumulation stage. During the first two stages, value increase of High Efficiency group is much more than that of Low Efficieny group, i.e. $102.94 \mathrm{mg} / \mathrm{kg}$ for KWS 8138 vs. $80.28 \mathrm{mg} / \mathrm{kg}$ for BETA176, which is in consistency with the peak value of ONUEp at vegetative stage. What's more, during each development stage, Soil Nitrogen Transformation Quantity of high efficiency genotype KWS8138 is significantly higher than that of low efficiency genotype BETA176.

Table 3. Transformation quantity of soil organic nitrogen at different developmental stages of Sugar beet (mg/kg).

\begin{tabular}{lllll}
\hline Genotypes & Seedling stage & Vegetative stage & Root and root increasing stage & Sugar accumulation stage \\
\hline KWS8138 (High Efficiency) & $77.21 \pm 25.33 \mathrm{a}$ & $180.15 \pm 35.21 \mathrm{a}$ & $244.03 \pm 28.76 \mathrm{a}$ & $321.24 \pm 30.20 \mathrm{a}$ \\
BETA176 (Low Efficiency) & $60.80 \pm 16.82 \mathrm{~b}$ & $141.08 \pm 29.64 \mathrm{~b}$ & $174.50 \pm 19.68 \mathrm{~b}$ \\
\hline
\end{tabular}

\subsection{Root-Shoot Ratio of Different Sugar Beet Genotypes}

The root-shoot ratio of sugar beet changes dramatically through its development stages. It decreases sharply from the seedling stage to the lowest level at the vegetative stage, and then increases gradually till sugar accumulation stage to its highest level (figure 2). Comparing the performance of two varieties in their growth season, we can see little difference prior to their vegetative stage, but since then, the ratio of variety KWS8138 is significantly higher than that of variety BETA176 (figure 2). 


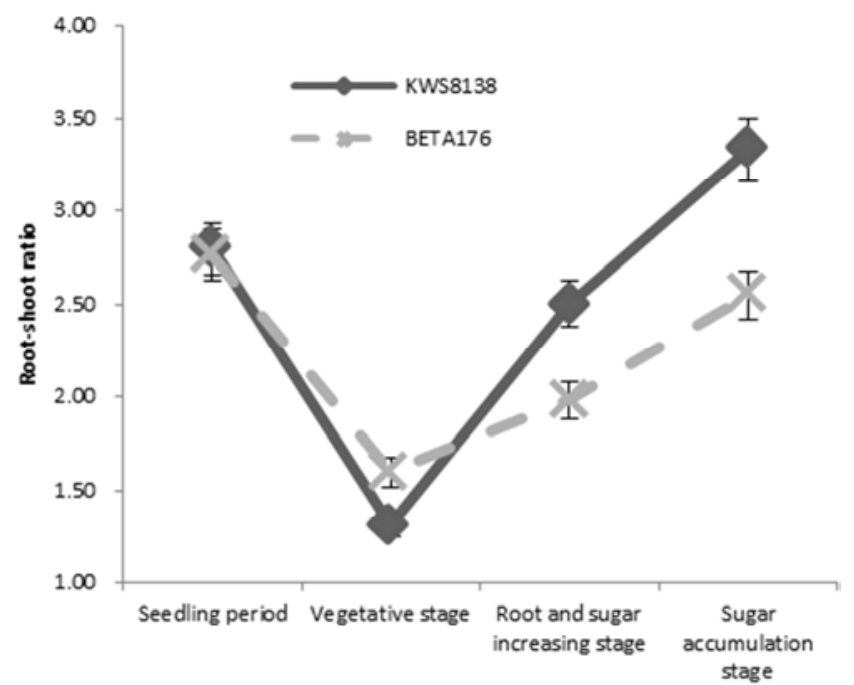

Figure 2. The root-shoot radio of sugar beet from seedling to sugar accumulation stage.

\section{Discussion}

Nitrogen efficiency covers both nitrogen use efficiency and nitrogen uptake efficiency [8]. Therefore, high nitrogen efficiency plants entail both high soil $\mathrm{N}$ uptake efficiency and high plant $\mathrm{N}$ use efficiency (nitrogen transformation and transportation) [11], which also applies to the case of soil organic nitrogen. Research results on 100 sugar beet genotypes indicate the relatively large $\mathrm{CV}$ of ONUE as well as its biological diversity.

Under such experimental condition, evaluation results of soil organic nitrogen use efficiency, based on dry matter productivity efficiency of either roots or whole plants, turn out to be consistent. Therefore, either of them qualifies as samples for screening in further experimental study.

Sugar beet genotypes with higher soil organic nitrogen use efficiency in seedling stage present in each of development stages larger soil organic nitrogen transfer amount and higher use efficiency, all reaching statistically significant level.

There is considerable difference in nitrogen uptake efficiency between high- and low-efficiency genotypes [6]. The determining factors in plants' nitrogen uptake ability are their root system's development and vitality [3], while minor factors being root biomass, root length and total root absorption area etc. [14, 5]. Therefore, during major development stages, good root morphology and vitality are fundamental elements for high nitrogen uptake and use efficiency [13]. In our research, the sugar beet genotypes with higher soil organic nitrogen transfer ability also exhibit higher root-shoot ratio, which further confirms that, in both sugar beet and all other plants, root system and growth vitality are crucial factors in higher soil organic nitrogen uptake efficiency. A proper root-shoot ratio is beneficial for maintaining a proper underground and aboveground root-shoot relationship, coordinating the distribution of nitrogen in each organ, stimulating a harmonious growth of underground and aboveground parts [12]. This might partially explain why sugar beet with high root-shoot ratio features high soil organic nitrogen use efficiency.

\section{Conclusion}

Research results on 100 sugar beet genotypes indicate the relatively large $\mathrm{CV}$ of ONUE, i.e. in aspect of soil ONUE sugar beet has a property of biological diversity.

To evaluate the property of a sugar beet genotype in soil ONUE, seedling stage is the best choice for rapid, accurate and economic aim.

Sugar beet dry matter productivity efficiency of either root or whole plant is suitable for screening in soil ONUE.

\section{Acknowledgements}

All authors acknowledge the National Natural Science Foundation of China (No. 31371686), Special in Soil and Fertilizer of Sugar Beet, Chinese Agricultural Research System (No. CARS-210306), Science and Technology Planning Project of Educational Department of Heilongjiang Province, China (No. 12541623).

\section{References}

[1] Abbasi MK, Khaliq A. 2016. Nitrogen mineralization of a loam soil supplemented with organic-inorganic amendments under laboratory incubation. Frontiers in Plant Science 7: 1038.

[2] Cong Yaohui, Zhang Yuling, Zhang Yulong et al. 2016. Soil Organic nitrogen components and their contributions to mineralizable nitrogen in paddy soil of the black soil region. ACTA PEDOLOGICA SINICA 2: 457-467.

[3] Dong G C, Chen C, Yuan Q M et al. 2016. The effect of nitrogen fertilizer treatments on root traits and nitrogen use efficiency in indica rice varieties with high nitrogen absorption efficiency. Acta Ecologica Sinica 3: 642-651.

[4] Jones DL, Kielland K, Sinclair FL, et al. 2009. Soil organic nitrogen mineralization across a global latitudinal gradient. Global Biogeochemical Cycles 23(1): n/a-n/a.

[5] Jiang Lin-lin, Han Li-Si, Han Xiao-Ri et al. 2011. Effects of nitrogen on growth, root morphological traits, nitrogen uptake and utilization efficiency of maize seedlings. Plant Nutrition and Fertilizer Science 1: 247-253.

[6] Li Shu-wen, Wen Hong-da, Zhou Yan-zhen et al. 2006. Characterization of nitrogen uptake and dry matter production in wheat varieties with different $\mathrm{N}$ efficiency. Scientia Agricultura Sinica 10: 1992-2000.

[7] Ma Dai, Li Fei, Meng Xian-yuan, et al. 2015. Progress on nitrogen nutrition of sugarbeet and diagnostic techniques. Sugar Crop of China 4: 56-58.

[8] Moll R H KEJ, Jackson W A. 1982. Analysis and interpretation of factors which contribute to efficiency of nitrogen utilization. Agronomy Journal 74(3): 3 .

[9] Reinhart KO, Cao X, Ma Q, et al. 2016. Elevational variation in soil amino acid and inorganic nitrogen concentrations in taibai mountain, china. Plos One 11(6): e0157979. 
[10] Roberts P, Bol R, Jones DL. 2007. Free amino sugar reactions in soil in relation to soil carbon and nitrogen cycling. Soil Biology and Biochemistry 39(12): 3081-3092.

[11] Sauerbeck DR, Helal HM. 1990. Factors affecting the nutrient efficiency of plants. In: El Bassam N, Dambroth M, Loughman BC, editors. Genetic Aspects of Plant Mineral Nutrition. Dordrecht: Springer Netherlands. p. 11-17.

[12] Wang Yan-Zhe, Liu Xiu-Wei, Sun Hong-Yong et al. 2013. Effects of water and nitrogen on root/shoot ratio and water use efficiency of winter wheat. Chinese Journal of Eco-Agriculture 3: 282-289.

[13] Wei Hai-Yan, Zhang Hong-Cheng, Zhang Sheng-Fei et al. 2008 Root morphological and physiological characteristics in rice genotypes withdifferent $\mathrm{N}$ use efficiencies. ACTA AGRONOMICA SINICA 3: 429-436.

[14] Xiong Shu-ping, Wu Ke-yuan, Wang Xiao-chun et al. 2016. Analysis of root absorption characteristics and nitrogen utilization of wheat genotypes with different $\mathrm{N}$ efficiency. Scientia Agricultura Sinica 12: 2267-2279.

[15] Zhang Yi-Fei, Zhang Xiao-xun, Liu Yang et al. 2013. Prospect of the sugar beet industrry in China. Heilongjiang Agricultural Sciences 8: 156-160.

[16] Zhou jian-Chao, Wang Qiu-Hong, Wang Xiao-Chun. 2014. Development of soil organic nitrogen mineralization and its influenced mechanisms to enlightenment of sugarbeet research. Sugar Crop of China 1: 65-67. 